\title{
Analysis of Various Features using Different Temporal Derivatives from Speech Signals
}

\author{
Muskan \\ PG Scholar, \\ Department of Computer Science and \\ Engineering, \\ Panjab University - UIET, Chandigarh
}

\author{
Naveen Aggarwal, Ph.D \\ Assistant Professor, \\ Department of Computer Science and \\ Engineering, \\ Panjab University - UIET, Chandigarh
}

\begin{abstract}
Speech recognition being an upcoming field is evaluated and research is being done for the same. Research in speech recognition for different languages is at peak. Less amount of work has been done for Indian languages particularly for Punjabi language. In this paper, Punjabi speech has been analyzed by extracting various features along with different temporal derivatives using feature extraction techniques. The dataset which has been considered for the research work is the set of Punjabi isolated digit recorded as 24 bit $44100 \mathrm{~Hz}$ mono PCM signal. Comparison of range and accuracy for acceptable results has been determined using HMM.
\end{abstract}

\section{Keywords}

Speech Recognition, MFCC, PLP, LPC, FBank, Melspec

\section{INTRODUCTION}

Speech is the source of communication. It carries data in the form of signals. Automatic Speech Recognition [1] (ASR) is the automatic speech transformation system which translates the audio input into text form. Identifying the words being spoken, mapping text to speech, verifying or identifying the speaker etc. are some of the activities being covered in speech processing. Although great amount of work has been performed in English language for speech recognition, Punjabi, being one of the most widely used languages, deserve to seek attention of researchers in terms of speech processing.

Accuracy, noise removal, information retrieval and varying bit rate are some of the most considerable part of speech recognition challenges. However, in this research paper, author has focussed on feature extraction of various speech signals of Punjabi language and its comparison. Also, the features have been analyzed based on different temporal derivatives.

\section{RELATED WORK}

Various other extraction techniques used are MFCC, LPC, PLP, Fbank and Melspec. These are used and are supported by HTK toolkit. All feature extraction techniques undergo usage of particular factors including delta coefficients, delta-delta coefficients, energy coefficients, absolute energy coefficients, $0^{\text {th }}$ coefficient, CRC checksum coefficient, compression coefficient etc. In 2001, Joseph Psutka et al. [26] performed experiments on comparison of MFCC and PLP parameters. Thereafter, H. Manabe et al. [28] proposed a model for EMG speech recordings of 10 Japanese words and extracting features thereby implementing Hidden Markov Model for the system. Various feature extraction techniques being implemented were FBANK, LPC, MFCC and LPCEPSTRA.

In 2005, Aik Ming Toh et al. [27] implemented speech recognition techniques MFCC and FBANK for different types of environments including additive, convolutive and reverberant. In 2010, Ravinder Kumar [22] worked on the comparison of Hidden Markov model and DTW techniques for isolated word recognition in Punjabi language. The author also states that if incase the size of codebook of HMM increases, the HMM recognition approach can become better but this may make the system more complex. Thus, it has been observed that the HTK and Audacity toolkit is used for feature extraction techniques. However, in Punjabi language, no such comparison has been performed on isolated words which can compare different features in presence of their temporal derivatives. Thus, five different features including MFCC, LPC, PLP, FBank and MelSpec are used in the presence of different temporal derivatives.

\section{LITERATURE SURVEY}

In 2013, Shweta Tripathi et al. [20] used HTK toolkit for Hindi Isolated word speech recognition. Feature extraction technique being used by them is LPC and MFCC. Results show that MFCC feature vector extraction technique has an edge over LPC feature extraction technique. Similar experiments were performed by Hong Quang et al [42] in 2010 by collecting speech samples for Vietnamese language from web and then framing bigram recognition model for the same. Basam A.Q. Al-Qatab et al [34] in 2010, performed HTK implementation for Arabic speech recognition.

Arabic word were recorded and converted to phonemes. Also, Ma Yahzhow et al [35] performed similar experiments with Russian speech but observed that HTK toolkit HMM recognition technique is not very efficient for speech recognition in Russian language as the dialects of the language vary from one group of people to the other. In 2011, A.N. Mishra et al [30] performed the experiments of speech recognition in HTK on connected Hindi words using MFCC and PLP feature extraction techniques along with some of its combinations. Finally, HMM was implemented. It has been observed that PLP feature extraction techniques along with its combinations perform better feature extraction techniques as compared to MFCC model which have been used traditionally.

In Jan 2014, SaiSujith Reddy Mankala et al [37] used HTK toolkit to implement the HMM speech recognition phase on Telghu language. In March 2014, K.Murali Krishna et al [39] used HMM for further speech recognition for Tamil words. They also used same method of MFCC along with HMM to implement speech recognition method on HTK toolkit. Ankit Kumar et al [38] proposed speech recognition system for MFCC and PLP feature extraction techniques based HMM recognition as per acoustic modelling in Hindi. 
Table 1: Summarization of research on Speech using HTK in different Arabic languages

\begin{tabular}{|c|c|c|c|c|c|}
\hline Year & Author & Algorithms & Input & Results & Language \\
\hline 2013 & $\begin{array}{l}\text { Shweta Tripathi et } \\
\text { al. [20] }\end{array}$ & $\begin{array}{l}\text { LPC, } \\
\text { HMM }\end{array}$ & Isolated Words & $\begin{array}{l}\text { Best: } \quad \text { Speaker } \\
\text { Dependent (MFCC) }\end{array}$ & Hindi \\
\hline 2010 & $\begin{array}{l}\text { Hong Quang et al } \\
\text { [42] }\end{array}$ & $\begin{array}{l}\text { MFCC, HMM, } \\
\text { MLLR }\end{array}$ & $\begin{array}{l}\text { Sentences recorded in } \\
\text { studio }\end{array}$ & $71.37 \%$ and $75.96 \%$ & Vietnamese \\
\hline 2010 & $\begin{array}{l}\text { Basam A.Q. Al- } \\
\text { Qatab et al [34] }\end{array}$ & MFCC, HMM & $\begin{array}{l}\text { Words and sentences } \\
\text { recorded }\end{array}$ & $97.99 \%$ accuracy & Arabic \\
\hline 2014 & $\begin{array}{l}\text { Ma Yahzhow et al } \\
{[35]}\end{array}$ & LPC, PLP, HMM & Words and Commands & $\begin{array}{l}\text { Better results Still } \\
\text { awaiting }\end{array}$ & Russian \\
\hline 2011 & $\begin{array}{l}\text { A.N. Mishra et al } \\
{[30]}\end{array}$ & $\begin{array}{l}\text { MFCC, PLP, } \\
\text { HMM }\end{array}$ & Isolated Words & $\begin{array}{l}\text { PLP is better than } \\
\text { MFCC }\end{array}$ & Hindi \\
\hline $\begin{array}{l}\text { Jan } \\
2014\end{array}$ & $\begin{array}{l}\text { SaiSujith Reddy } \\
\text { Mankala et al [37] }\end{array}$ & MFCC, HMM & $\begin{array}{l}\text { Data recordings by Sony } \\
\text { F-V120 }\end{array}$ & $96 \%$ of accuracy & Telghu \\
\hline $\begin{array}{l}\text { Mar } \\
2014\end{array}$ & $\begin{array}{l}\text { K.Murali Krishna et } \\
\text { al [39] }\end{array}$ & $\begin{array}{l}\text { MFCC, } \\
\text { HMM }\end{array}$ & $\begin{array}{l}\text { Recordings in living room } \\
\text { at } 16 \mathrm{kHz}\end{array}$ & $84 \%$ & Tamil \\
\hline 2014 & $\begin{array}{l}\text { Ankit Kumar et al } \\
\text { [38] }\end{array}$ & $\begin{array}{l}\text { MFCC, } \\
\text { HMM }\end{array}$ & Connected Hindi Words & $95.08 \%$ accuracy & Hindi \\
\hline
\end{tabular}

\section{EXPERIMENTAL SETUP}

Speech has been recorded at 24 bit $44100 \mathrm{~Hz}$ for each digit of Punjabi. However, in recordings for this research work, it has been observed that before pronouncing any word and after pronunciation of word, silence is observed. Different Punjabi isolated digits are shown in Table 1

For each speech signal, five different features have been extracted:

- Mel Frequency Cepstrum Coefficients

- $\quad$ Linear Predictive Coefficients

- Perceptual Linear Predictions

- $\quad$ Filter banks

- Mel spectrum

\begin{tabular}{|l|l|l|}
\hline Word & English & Punjabi \\
\hline 0 & Zero & Sifar \\
\hline 1 & One & Ik \\
\hline 2 & Two & Do \\
\hline 3 & Three & Tin \\
\hline 4 & Four & Char \\
\hline 5 & Five & Panj \\
\hline 6 & Six & Chhe \\
\hline 7 & Seven & Satt \\
\hline 8 & Eight & Ath \\
\hline 9 & Nine & Nau \\
\hline
\end{tabular}

Table 2: Different Punjabi digits

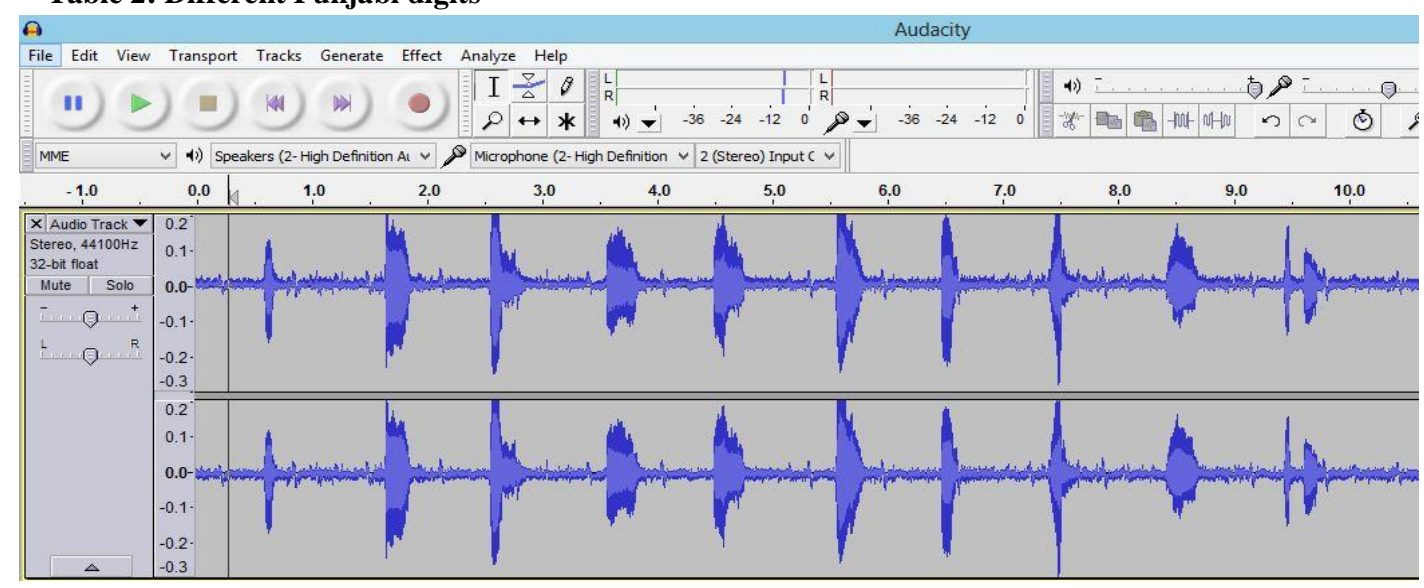

Fig. 1: Punjabi speech signals: ik do tinn char panj chhe satt ath nau sifar

It has been observed that before signal and after signal there is silence as there is very low amplitude. Also, in between two strong variations, there exists another low amplitude part which is not silence. Thus, every low amplitude signal is not silence.

Finally, for each feature extraction technique, different combinations of temporal derivatives which are used are: 
- Delta + Acceleration Coefficients

- Delta Coefficients

- $\quad$ Energy + Delta Coefficients

- $\quad$ None

Here, there is need to understand temporal derivatives. Some major temporal derivatives being used are as follows:

- Delta Coefficients: Delta coefficients are the first order derivatives of basic coefficients of MFCC features being extracted.

$$
\mathrm{d}_{\mathrm{t}}=\sum_{\mathrm{n}=1}^{\mathrm{N}} \mathrm{n}\left(\mathrm{c}_{\mathrm{t}+\mathrm{n}}-\mathrm{c}_{\mathrm{t}-\mathrm{n}}\right) / 2 \sum_{\mathrm{n}=1}^{\mathrm{N}} \mathrm{n}^{2}
$$

- Delta - Delta Coefficients: Delta coefficients are the second order derivatives of basic coefficients of MFCC features being extracted. Or these can be defined as first order derivatives of delta coefficients. The higher order derivative of coefficients comparatively has less numerical value. For this, these are re-sampled using CEPLIFTER.
- Energy Coefficients: Energy coefficients refer to those coefficients which indicate the energy calculation of the signal at various chunks of frequencies uttered by speaker.

The Punjabi dataset of isolated words which are being used has been shown in fig 1. It indicates all those digits which are considered during collection of training data. In order to perform this experiment, HTK toolkit was used along with Audacity tool. Hidden Markov Model was used to perform these experiments.

\section{OBSERVATIONS}

This experiment has been performed on data set of 100 speech samples at 24 bit $44100 \mathrm{~Hz}$ which have been taken in real time environment of a living room. Different feature extraction techniques are used in combination with delta and acceleration coefficients. As per analysis using MATLAB, it has been observed that upper bound and lower bound for every feature extraction technique differs. The table 2 shows the similar results.

Table 3: Observations of Lower and Upper Bounds for different feature extraction techniques for word "sifar"

\begin{tabular}{|l|l|l|}
\hline Speech Feature Extraction Techniques & \multicolumn{2}{|l|}{ Range } \\
\cline { 2 - 3 } & Lower Bound & Upper Bound \\
\hline MFCC_D_A & -40 & 20 \\
\hline
\end{tabular}




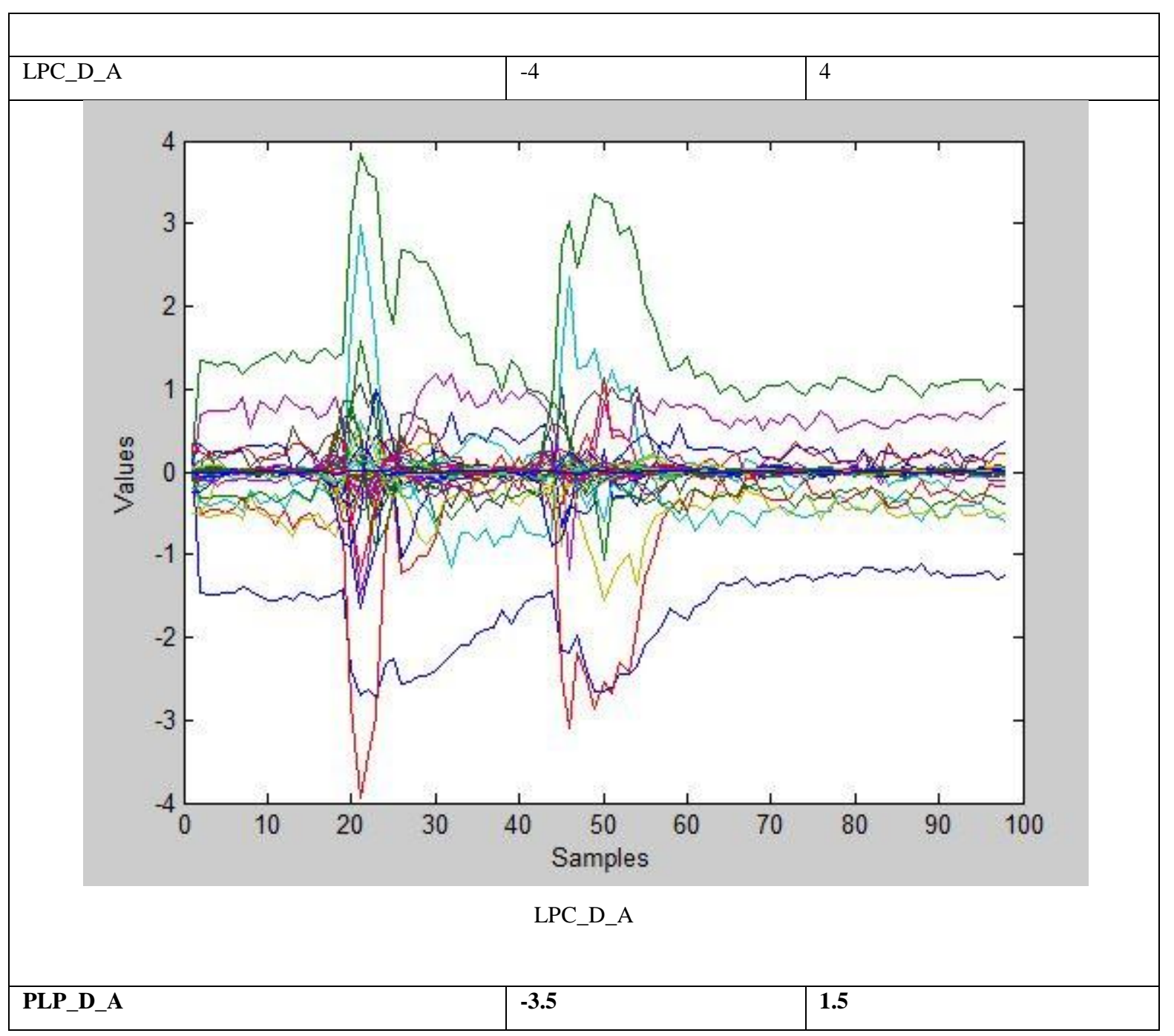




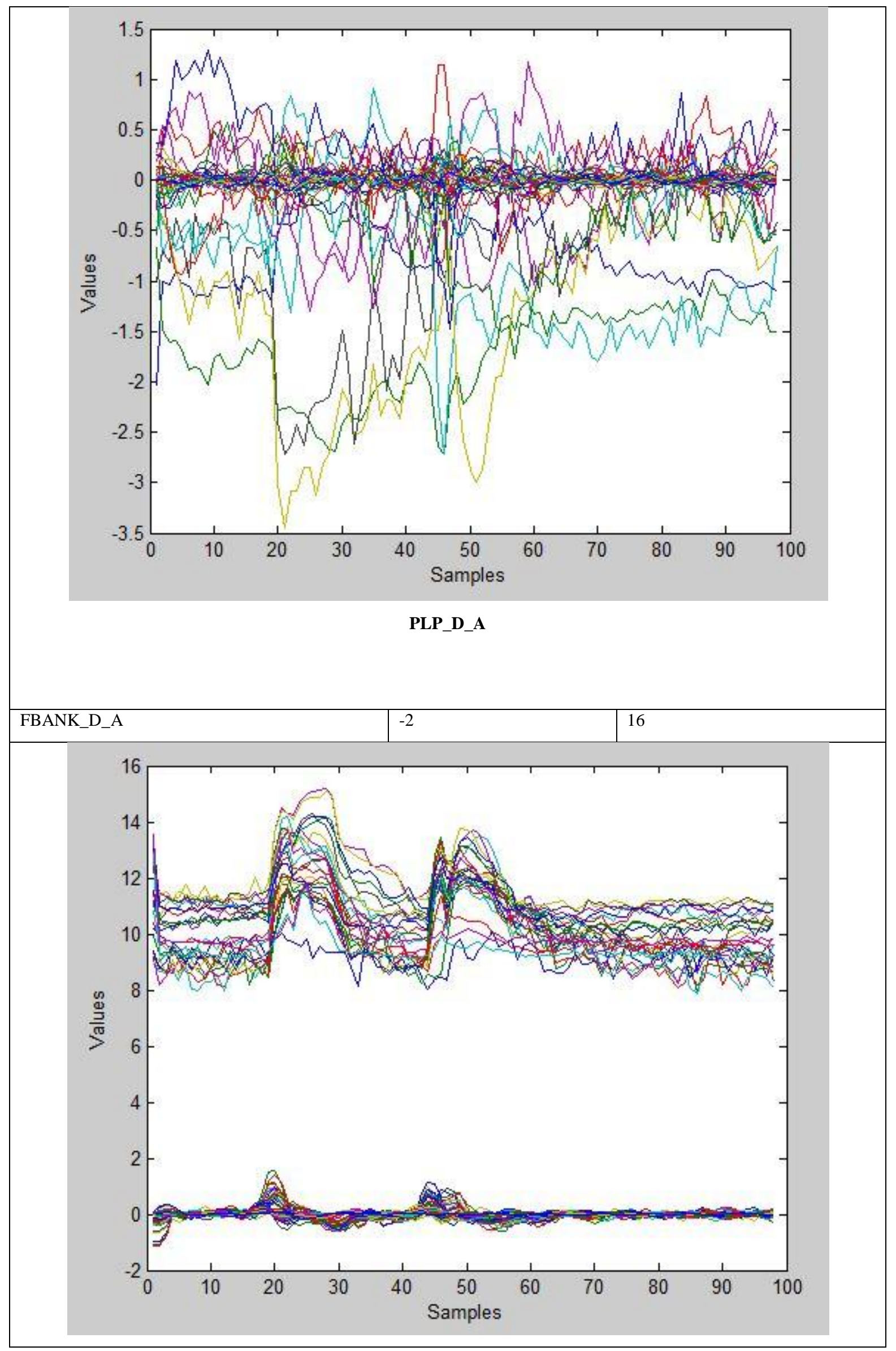




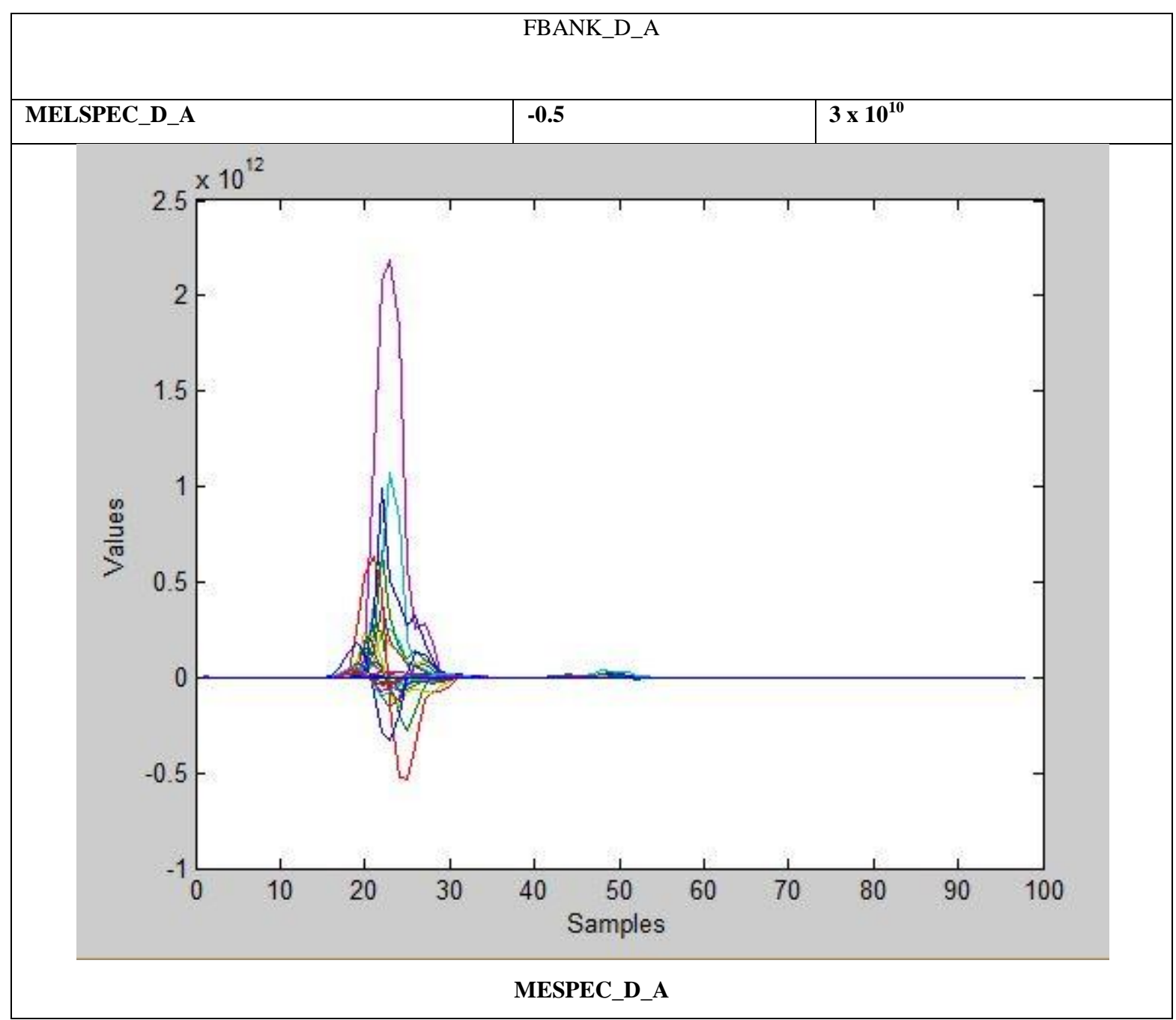

The observation for 24 bit $44100 \mathrm{~Hz}$ testing samples on the given speech corpus have been observed and different outputs have been recorded. These results have been shown in tabular format in Table 3. All the speech samples have been recorded in real time environment using Audacity toolkit as a mono 24 bit $44100 \mathrm{~Hz}$ PCM signal .

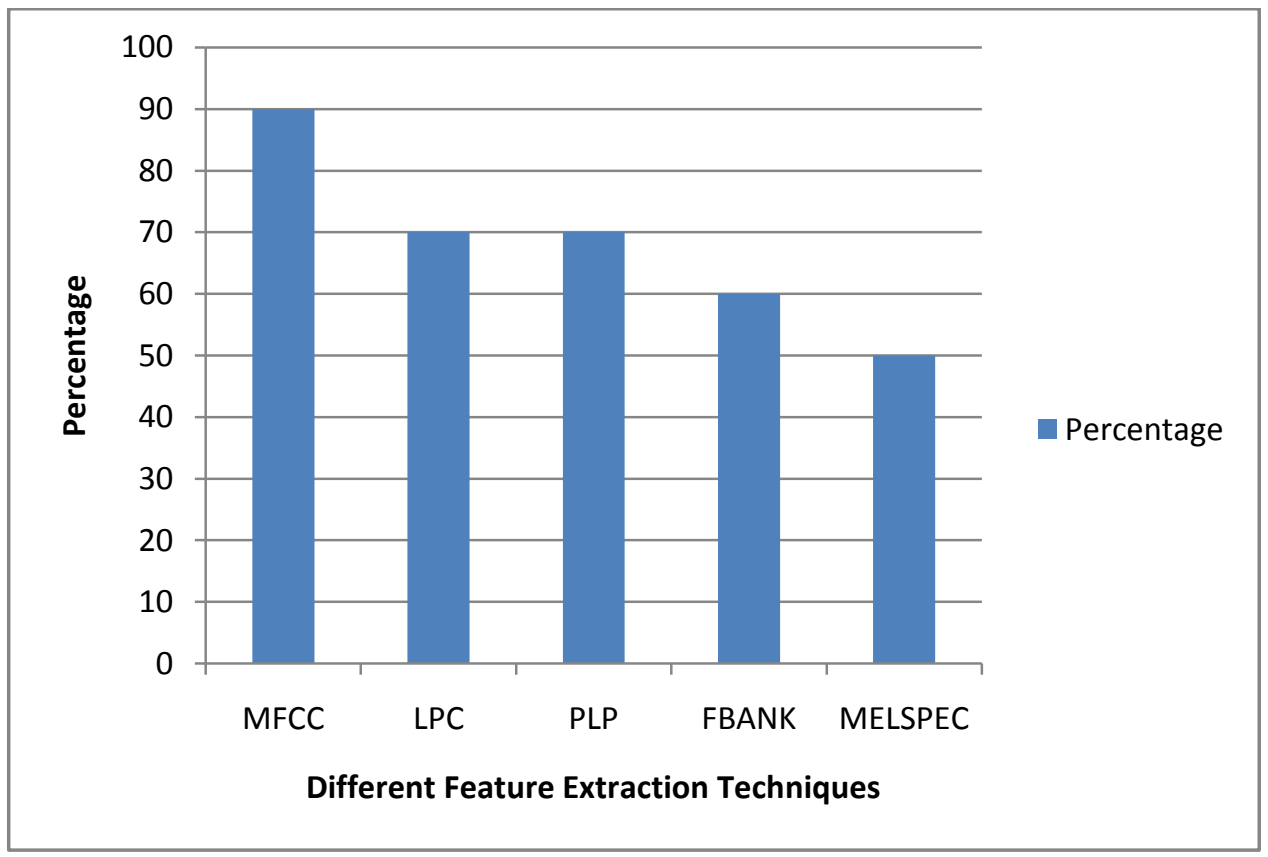

Fig 2: Analysis of different feature extraction techniques 
Table 4: Testing of samples on speech corpus recorded in vacant room

\begin{tabular}{|l|l|l|l|l|l|}
\hline Digit & MFCC & LPC & PLP & FBANK & MELSPEC \\
\hline Ik & Correct & Correct & In-Correct & Correct & In-Correct \\
\hline Do & Correct & In-Correct & Correct & Correct & In-Correct \\
\hline Tin & In-Correct & Correct & In-Correct & Correct & In-Correct \\
\hline Chaar & Correct & In-Correct & Correct & In-Correct & Correct \\
\hline Panj & Correct & In-Correct & Correct & In-Correct & Correct \\
\hline Chhe & Correct & Correct & Correct & Correct & Correct \\
\hline Satt & Correct & Correct & Correct & In-Correct & In-Correct \\
\hline Ath & Correct & Correct & Correct & Correct & Correct \\
\hline Nau & Correct & Correct & In-Correct & Correct & In-Correct \\
\hline Sifar & Correct & Correct & Correct & In-Correct & Correct \\
\hline Percentage & $90 \%$ & $70 \%$ & $70 \%$ & $60 \%$ & $50 \%$ \\
\hline
\end{tabular}

Table 5: Testing of samples on speech corpus

\begin{tabular}{|l|l|l|l|l|}
\hline Digit & MFCC & MFCC_D & MFCC_D_A & MFCC_E_D \\
\hline Ik & Correct & Correct & Correct & Correct \\
\hline Do & Correct & Correct & Correct & Correct \\
\hline Tin & In-Correct & Correct & Correct & Correct \\
\hline Chaar & Correct & Correct & Correct & In-Correct \\
\hline Panj & Correct & Correct & Correct & Correct \\
\hline Chhe & Correct & Correct & Correct & Correct \\
\hline Satt & In-Correct & Correct & Correct & Correct \\
\hline Ath & Correct & In-Correct & Correct & Correct \\
\hline Nau & Correct & Correct & Correct & Correct \\
\hline Sifar & Correct & Correct & Correct & $90 \%$ \\
\hline Percentage & $80 \%$ & $90 \%$ & $100 \%$ & \\
\hline
\end{tabular}

Finally, for given dataset as observed in fig 2, in terms of betterment of accuracy in decreasing order,

\section{MFCC $>$ LPC $>$ FBANK $>$ PLP $>$ MELSPEC.}

This clearly indicates that MFCC_D_A (MFCC along with delta and acceleration coefficients) is the best recognition technique whereas MFCC is the least accurate one. There are many different temporal derivatives for each of the feature extraction techniques. However, in this work, only few temporal derivatives have been used.

This is performed in order to analyze the speech at different circumstances to get the best recognition technique. This study is recognized for MFCC_D, MFCC_D_A, MFCC_E_D and MFCC. However, it has been analyzed with the speech signals of more samples. This can be observed in Table 5. Thus, accuracy in decreasing order is

\section{MFCC_D_A > MFCC_E_D > MFCC_D > MFCC}

Various experiments have been performed on other feature extraction techniques for different temporal derivative. It has been observed that same pattern is observed in terms of temporal derivatives for decreasing order of accuracy in other techniques as well.

\section{CONCLUSION}

This research can be summarized to find the best feature extraction techniques for isolated digits of Punjabi speech signals. MFCC is the best feature extraction techniques among MFCC, LPC, PLP, MELSPEC, FBANK. Also, it has been observed that MFCC_D_A temporal derivative performs the best performance in comparison to all other temporal derivatives.

These results have been observed when voices are recorded in vacant room where no other speech signals/ noise participated. Although, the real time recordings have been taken using HP microphone and Audacity tool, the observations may vary for other toolkit. This research has been carried out using HMM. However, other different algorithms like Deep Neural Network, Genetic algorithms etc. can be applied for the same. Also, the speech corpus is limited. Hence, large speech corpus may change the results. Different bit rates may affect results which have been obtained in this research work. 
Further, this research work can be improved and worked upon for gender recognition, speaker identification by using the optimum temporal derivatives along with best feature extraction technique. Considering different samples of population for this analysis is good path of research since this research is based on Punjabi language which is tonal. Further analysis on continuous Punjabi speech recognition can be made which can contain different dialects. Based on the geographical regions of different parts of north India and Pakistan, different form of dialects can be considered.

\section{REFERENCES}

[1] L. Rabiner and R. Schafer, "Introduction to digital speech processing", Foundations and Trends in Signal Processing, Journal of ACM vol. 1, no. 1-2, pp. 1-194, 2007.

[2] L. Rabiner and B. H. Jaung, Fundamentals of Speech Recognition, Englewood Cliffs, NJ: Prentice-Hall, 1993.

[3] X. Huang, J. Baker and R Reddy, "A Historical Perspective of Speech Recognition", Communications of the ACM, vol. 57, no. 1, January 2014.

[4] K. H. Davis, R. Biddulph and S. Balashek, "Automatic recognition of spoken digits," J.A.S.A., vol. 24, no. 6, pp. 637-642, 1952

[5] S. C. Sajjan and C. Vijaya, "Comparison of DTW and HMM for isolated word recognition", Proceedings of International Conference on Pattern Recognition, Informatics and Medical Engineering (PRIME), IEEE, pp. 466-470, 2012.

[6] H Sakoi and S Chiba, "Dynamic Programming Algorithm Optimization for Spoken Word Recognition", IEEE Transactions on acoustics, speech and signal processing, vol. Assp- 26, no. 1, February 1978.

[7] L R Rabiner, A E Rosenberg, S E Levinson and J G Wilpon, "Speaker-Independent Recognition of Isolated Words Using Clustering Techniques", IEEE Transactions on acoustics, speech and signal processing, vol. Assp- 27, no. 4, August 1979.

[8] L. R. Rabinar and M. R. Sambur, "An algorithm for determining the endpoints of isolated utterances", The Bell System Technical Journal, pp. 297-315, 1975.

[9] L R Rabiner, A E Rosenberg, L F Lamel and J G Wilpon , "An Improved Endpoint Detector for Isolated Word Recognition", IEEE Transactions on acoustics, speech and signal processing, vol. Assp- 29, no. 4, 1981.

[10] M A Bush, G E Kopec and N Lauritzen, "Segmentation in Isolated Word Recognition Using Vector Quantization", Acoustics, Speech, and Signal Processing, IEEE International Conference on ICASSP '84, vol. 9, 1984

[11] L R Rabiner and B H Jaung, "An Introduction to Hidden Markov Models", IEEE ASSP Magazine, pp. 4-16, January 1986.

[12] L R Rabiner and B H Jaung, "Hidden Markov Models for Speech Recognition", Technometrics, vol 33, no. 3, 1991.

[13] S B Davis and P Mermelstein, "Comparison of Parametric Representations for Monosyllabic Word Recognition in Continuously Spoken Sentences", IEEE
Transactions on acoustics, speech and signal processing, vol. Assp- 28, no. 4, 1980.

[14] Wei Han, Cheong-Fat Chan, Chiu-Sing Choy and KongPang Pun, "An Efficient MFCC Extraction Method in Speech Recognition", Proceedings of IEEE, ISCAS 2006, 2006

[15] H A Patil and T K Basu, "Development of speech corpora for speaker recognition research and evaluation in Indian languages", IJST 2008, Springer, 2008

[16] S Ranjan, "A Discrete Wavelet Transform Based Approach to Hindi Speech Recognition", Proceedings of International Conference on Signal Acquisition and Processing, IEEE, pp, 345-348, 2010.

[17] K S Rao, "Application of prosody models for developing speech systems in Indian languages", IJST 2011, Springer, 2011.

[18] I Bhardwaj and N D Londhe, "Hidden Markov Model Based Isolated Hindi Word Recognition", Proceedings of 2nd International Conference on Power, Control and Embedded Systems, IEEE, 2012.

[19] T Pruthi, S Saksena and P K Das, "Swaranjali: Isolated Word Recognition for Hindi Language using VQ and HMM", International Conference on Multimedia Processing and Systems (ICMPS), IIT Madras.

[20] S Tripathy, N Baranwal and G C Nandi, "A MFCC based Hindi Speech Recognition Technique using HTK Toolkit", Proceedings of the 2013 IEEE Second International Conference on Image Information Processing (ICIIP-2013), pp. 539-544, 2013.

[21] A Sharma and A Kaur, "Automatic Segmentation of Punjabi Speech into Syllable-Like Units using Group Delay: A Review", Proceedings of International Journal of Computer Science \& Engineering Technology (IJCSET), vol 4, no 6, 2013.

[22] R. Kumar, "Comparison of HMM and DTW for isolated word recognition system for punjabi language", Proceedings of IJSC, vol 5, no. 3, pp. 88-92, 2010

[23] Gurpreet Kaur, Parminder Singh and Amandeep Kaur, "Syllable Boundary Detection System for Punjabi Language", Proceedings of International Journal of Applied Research in Computing, vol.1, no. 2, July 2013.

[24] Ramana Rao G. V. and Srichand J, "Word boundary detection using pitch variations", Proceedings of Fourth International Conference on Spoken Language, 1996. ICSLP 96, pp. 813-816, May 1996.

[25] Wiqas Ghai and Navdeep Singh, "Continuous Speech recognition for Punjabi Language", International Journals of Computer Application, vol. 72, no. 14, May 2013.

[26] J. Psutka, L. Muller and J. V. Psutka, "Comparison of MFCC and PLP Parametrizations in Speaker Independent continuous speech recognition task", Eurospeech 2001, Scandanavia.

[27] A. M. Toh, R. Togneri and S. Nordholm, "Investigating robust features for speech recognition in hostile environment", Asia Pacific Conference on Communication IEEE, October 2005. 
[28] H. Manabe and Z. Zhang, "Multi-stream HMM for EMG-Based Speech Recognition", Multimedia Laboratories, NTT Docomo, Kanagawa, Japan.

[29] Muskan and Naveen Aggarwal, "Punjabi Speech Recognition: A Survey", Proceedings of ICAET, May 2014.

[30] A. N. Mishra, M Chandra, A Biswas, S. N. Sharan, "Robust features for connected Hindi digits recognition", International Journal of Signal Processing, Image Processing and Pattern Recognition, Vol. 4, No. 2, June, 2011

[31] K. M Krishna, M V Lakshmi and S. Sathiya Lakshmi, "Feature Extraction and Dimensionality Reduction using IPS for Isolated Tamil Words Speech Recognizer", International Journal of Advanced Research in Computer and Communication Engineering, Vol. 3, Issue 3, March 2014.

[32] M Alsulaiman, G Muhammad and Z Ali, "Comparison of Voice Features for Arabic Speech Recognition", IEEE, 2011.

[33] V Tiwari, "MFCC and its applications in speaker recognition", Proceedings of IJET, 2010.

[34] Bassam A. Q. Al-Qatab and Raja N. Ainon, “Arabic speech recognition using Hidden Markov Model toolkit (HTK)", IEEE, 2010.

[35] M Yanzhou and Y Mianzhu, "Russian Speech Recognition System Design Based on HMM", Proceedings of LEMCS, 2014.
[36] J Kaur, Nidhi, R Kaur, "Issues involved in speech-to-text conversion", International Journal Of Computational Engineering Research, Vol. 2, Issue No.2, Page No. 512515, Mar-Apr 2012

[37] S R Mankala, S R Bojja, V S Ramaiah \& R. Rajeswara Rao, "Automatic speech processing using HTK for Telghu language", International Journal of Advances in Engineering \& Technology, Jan. 2014

[38] A Kumar, M Dua and T Chaudhary, "Continuous Hindi Speech Recognition using Monophone based Acoustic Modeling", International Journal of Computer Applications, 2014.

[39] K. Murali Krishna, M. Vanitha Lakshmi and S. Sathiya Lakshmi, "Feature Extraction and Dimensionality Reduction using IPS for Isolated Tamil Words Speech Recognizer", International Journal of Advanced Research in Computer and Communication Engineering, Vol. 3, Issue 3, March 2014

[40] S Young, "The HTK Book", Cambridge University Engineering Department.

[41] E Vozarikova, J Juhar and A Cizmar, "Dual Shots Detection", Information and Communication technologies and services, Vol. 10, Issue. 4, 2012.

[42] N H Quang, T V Loan, LE The Dat, "Automatic Speech Recognition for Vietnamese using HTK System”, IEEE, 2010. 